\title{
PERFIL HORMONAL SEXUAL DE MULHERES EM PROGRAMA CRÔNICO DE DIÁLSE EM TRATAMENTO COM ERITROPOETINA HUMANA RECOMBINANTE
}

\author{
Rosina Conceição Graçaplena Dal Maso*, Mário Cavagna Neto, luis Yu, Yara Juliano, \\ Neil Ferreira Novo, Maria Cristina Cury, Miguel Bogossian \\ Resumo de tese de Mestrado apresentada à Faculdade de Medicina de Santo Amaro, na \\ área de Saúde Materno Infantil, São Paulo, SP.
}

RESUMO - A IRC (insuficiência renal crônica) terminal é acompanhada por alterações sistêmicas, inclusive do eixo hipotálamohipofisário-gonadal. Após o aparecimento da EPO (eritropoetina humana recombinante), surgiram relatos de melhora da disfunção sexual em homens, havendo relativa escassez de dados sobre dosagens hormonais e função sexual das mulheres. No Brasil, há poucos dados sobre o perfil hormonal de mulheres em programa crônico de diálise.

OBjetivos. Avaliação e comparação do perfil hormonal sexual, através das dosagens de FSH (hormônio folículo estimulante), LH (hormônio luteinizante), PRL (prolactina) e E2 (estradiol) de muIheres em programa crônico de diálise, sob terapêutica com EPO.

Métodos. Foram avaliadas 47 mulheres (uma em diálise peritoneal intermitente, quatro em diálise peritoneal ambulatorial contínua e 42 em hemodiálise), subdivididas em três grupos: (I) Grupo I, de mulheres com menos de 48 anos de idade e menstruando regularmente; (2) Grupo II, de mulhe- res com menos de 48 anos de idade e amenorreicas; (3) Grupo III, de mulheres com mais de 48 anos de idade e amenorreicas. Os grupos foram comparados entre si através do teste de Mann-Whitney para duas amostras independentes.

RESULTADOS. Todos os grupos mostraram valores normais das dosagens séricas de FSH, LH e E2, e não houve diferença estatisticamente significante nos níveis desses hormônios entre as mulheres que menstruam e as amenorreicas com idade inferior a 48 anos. Os níveis de PRL encontravam-se acima da normalidade em todos os grupos, independentemente da idade e do padrão menstrual, não havendo diferenças estatísticas significantes entre os grupos. As pacientes do grupo III apresentaram perfis hormonais compativeis com a menopausa, ou seja, níveis séricos de FSH e LH elevados e de E2 diminuídos.

UNITERMOS: Insuficiência renal crônica terminal. Diálise. Hormônios sexuais. Mulheres. Eritropoetina humana recombinante.

\section{INTRODUÇÃO}

Antes do advento da eritropoetina humana recombinante (EPO), a anemia era comum e responsabilizada por várias alterações presentes na insuficiência renal crônica terminal (IRCT), inclusive as de ordem sexual. Sua correção por transfusões repetidas implicava em risco aumentado de infecções por vírus das hepatites ${ }^{1,2}$, oferta excessiva de antígenos de histocompatibilidade ${ }^{3}$, etc. A disfunçãosexual, a infertilidade e a diminuição da libido eram amplamente conhecidas em homens e mulheres, e estas apresentavam alterações menstruais, classicamente, amenorréia ${ }^{4,5}, 0$ que levou à publicação de vários estudos de dosagens hormonais no final dos anos $70 \mathrm{e}$ início dos anos 804,6,7,8,9,10,11. O uso clínico da EPO permitiu o tratamento adequado, eficaz

* Correspondência:

Rua José da Silva Rainho 636- 14

Guarujá - São Paulo

CEP: $11420-390$ e sem os riscos descritos da anemia, além de melhora da qualidade de vida ${ }^{2,12,13,14}$ e da função sexual de homens e mulheres ${ }^{12,13,15}$. Desde então, existem na literatura inúmeros relatos sobre a melhora da impotência em homens, havendo comparativamente uma escassez de dados sobre o perfil hormonal sexual das mulheres em programa crônico de diálise. Em nosso meio, os dados são praticamente inexistentes.

Com o advento da EPO e o consequente tratamento da anemia, apareceram relatos de normalização dos níveis de PRL e retorno da menstruação ${ }^{12,13}$, bem como supressão dos níveis basais de $\mathrm{FSH}$ e $\mathrm{LH}^{15}$, com os autores sugerindo que os pacientes tratados com EPO apresentavam melhora da função sexual por normalização da retroalimentação no eixo hipotálamo-hipofisário-gonadal, já que pacientes com os mesmos níveis de hemoglobina e hematócrito sem EPO apresentavam perfis hormonais alterados. Os mesmos resultados não foram obtidos por outros autores ${ }^{33} \mathrm{e} \mathrm{um}$ estudo comparando pacientes policitêmicos (com baixa EPO endógena) com pacientes anêmicos (com alta EPO endógena) sugeriu que a doença de base, mais do que os níveis de EPO, é que realmente afeta os diversos eixos hormonais (em especial, o eixo hipotálamohipofisário), embora o tratamento da anemia, per se, possa melhorar alguns parâmetros hormonais ${ }^{34}$.

Os estudos relatados geralmente comparam os perfis das pacientes renais crônicas em diálise com controles normais ou receptoras de transplante renal. Este estudo pretende avaliar e comparar o perfil hormonal de mulheres que menstruam com mulheres amenorreicas, todas em programa de diálise e em tratamento coma EPO, através da comparação dos níveis séricos de FSH, LH e E2 de mulheres em programa crônico de diálise que menstruam e amenorreicas, com idade inferior a 48 anos; do padrão hormonal da PRL de pacientes em programa crônico de diálise que menstruam, amenorreicas com idade menor 
que 48 anos e amenorreicas com idade igual ou superior a 48 anos e se os níveis séricos de FSH, LH e E2 de pacientes amenorreicas e com idade igual ou superior a 48 anos, em programa crônico de diálise, são compatíveis com o perfil hormonal da menopausa

\section{Métodos}

Foram estudadas todas as mulheres em programa crônico de diálise (47) na Unidade de Diálise do Hospital Escola Wladimir Arruda, no período de I de maio a 3 I de dezembro de 1999, divididas nos seguintes grupos:

- Grupo I: mulheres que menstruam

- Grupo II: mulheres amenorreicas com idade inferior a 48 anos

- Grupo III: mulheres amenorreicas com idade superior ou igual a 48 anos

Utilizou-se, arbitrariamente, o limite etário de 48 anos como a idade divisória entre pacientes no menacme e na menopausa. Esse limite foi estabelecido com base no trabalho de Halbe et al. ${ }^{16}$, que mostrou que a média étaria da menopausa em nosso meio é de 47.8 anos, aumentando discretamente para 48 anos quando se separam as pacientes que tiveram menopausa cirúrgica.

Todas as mulheres encontravam-se em programa crônico de diálise, sendo quatro sob diálise peritoneal ambulatorial contínua, uma sob diálise peritoneal intermitente e 42 sob hemodiálise três vezes por semana, com sessões de três horas e meia, utilizando-se rim artificial de fibras ocas com membrana de polissulfona ( $\mathrm{F} 6$ - Fresenius ${ }^{\mathrm{TM}}$ ), e recebendo eritropoetina humana recombinante (Eprex $\AA$ ou Hemax $\left.{ }^{\circledR}\right)$ nas doses ajustadas às suas necessidades (3000 Ul a I 2000 Ul por semana).

No Grupo I, coletou-se sangue das mulheres para dosagens de FSH, LH, PRLeE2 (estradiol) no segundo ou terceiro dia do ciclo menstrual; nos Grupos II e III realizaram-se as dosagens de FSH, LH, PRL e E2 em uma das sessões de diálise. As pacientes em DPAC e DPI tiveram seu sangue coletado juntamente com a rotina mensal de exames, levando-se em consideração que todas elas eram amenorreicas.

As dosagens hormonais de LH, FSH, PRLe E2 foram realizadas em duplicata e pelo método ELISA, no aparelho ETI STAR - Dia Sorin no Laboratório de Análises Clínicas do Hospital Escola Wladimir Arruda, usando-se os seguintes kits:

Tabela I - Médias e medianas das idades (anos), do tempo de diálise (anos), e dos níveis de hemoglobina (g/dL), hematócrito (\%), FSH (mUl/ml), LH ( $\mathrm{mUl} / \mathrm{ml}), \mathrm{PRL}(\mathrm{ng} / \mathrm{ml})$ e estradiol $(\mathrm{pg} / \mathrm{ml})$ de mulheres com menos de 48 anos que menstruam (Grupo l) ou não menstruam (Grupo II) e de mulheres com mais de 48 anos (Grupo III)

\begin{tabular}{|c|c|c|c|}
\hline & GRUPOI & GRUPO II & GRUPO III \\
\hline Médiaidade & 32,81 & 34,78 & 60,17 \\
\hline Médiatempodiálise & 1,57 & 1,07 & 3,06 \\
\hline Medianahemoglobina & 9,9 & 9,9 & 9,2 \\
\hline Medianahematócrito & 31,5 & 29 & 29 \\
\hline MedianaFSH & 7,03 & 14,50 & 112,85 \\
\hline MedianaLH & 3,40 & 6,20 & 72,80 \\
\hline MedianaPRL & 80,20 & 165,40 & 65,10 \\
\hline Medianaestradiol & 59,27 & 53,70 & 20,83 \\
\hline
\end{tabular}

Valores da normalidade: $\mathrm{FSH}=0,5 \mathrm{a} 37,3 \mathrm{mUl} / \mathrm{ml}$ (não menopausadas),

$\mathrm{FSH}=42,3$ a $187,6 \mathrm{mUI} / \mathrm{ml}$ (menopausadas), $\mathrm{LH}=0,6 \mathrm{a} 96,2 \mathrm{mUI} / \mathrm{ml}$ (não menopausadas), $\mathrm{LH}=8,4$ a $102,2 \mathrm{mUI} / \mathrm{ml}$ (menopausadas), PRL $=8,39 \mathrm{a} 20,15 \mathrm{ng} / \mathrm{ml}$, estradiol $=30 \mathrm{a} 120 \mathrm{pg} / \mathrm{ml}$ (fasefolicular), estradiol $=15 \mathrm{a} 60 \mathrm{pg} / \mathrm{ml}$ (menopausadas)

Teste de Mann-Whitney para os níveis de FSH

I) Grupo IX Grupo II

$z$ calculado $=1,55$ (n.s.)

$\left.2^{\circ}\right)$ Grupo IIX Grupo III

$z$ calculado $=2,88^{*}(p<0,01)$

Teste de Mann-Whitney para os níveis de LH

$\left.1^{\circ}\right)$ Grupo IX Grupoll

$z$ calculado $=1,05$ (n.s.)

$2^{\circ}$ ) Grupo IIX Grupo III

$z$ calculado $=2,57^{*}(p<0,01)$

- LH, FSH e PRL: Genzyme Diagnostics Medix Biotech-ELISA

- Estradiol: Diatech Estradiol-I.I.D-ELISA

\section{Método estatístico}

Para a análise dos resultados foram utilizados testes não-paramétricos, considerando-se a natureza das variáveis ou a variabilidade das medidas efetuadas.

Aplicou-se o teste de Mann-Whitney para duas amostras independentes ${ }^{35}$, com o objetivo de comparar, dois a dois, os três grupos estudados, levando-se em conta a comparabilidade dos grupos: foram feitas comparações entre o Grupo I versus Grupo Il e entre Grupo II versus Grupo III.

Em todos os testes fixou-se em 0,05 ou $5 \%$, o nível para a rejeição da hipótese de nulidade.

\section{Resultados}

As médias das idades, do tempo de diálise, e as medianas dos níveis de hemoglobina, hematócrito, FSH, LH, PRL e estradiol das mulheres de cada grupo estão representadas na Tabela I.

\author{
Teste de Mann-Whitney para os níveis de PRL \\ 1') Grupo IX Grupo II \\ $\mathrm{z}$ calculado $=0,50$ (n.s.) \\ 2) Grupo IIXGrupo III \\ $\mathrm{z}$ calculado $=0,91$ (n.s.) \\ Teste de Mann-Whitney para estradiol \\ 19) Grupo IX Grupo II \\ z calculado $=0,59$ (n.s.) \\ 2) Grupo IIX Grupo III \\ $\mathrm{z}$ calculado $=0,72$ (n.s.)
}

Não houve diferença estatística em nenhum dos paramêtros estudados entre os Grupos I ell (mulheres com menos de 48 anos que menstruam versus mulheres com menos de 48 anos amenorrêicas). Na comparação entre os Grupos II e III (mulheres amenorreicas com menos de 48 anos versus mulheres amenorreicas com mais de 48 anos) houve diferença significante entre os níveis de FSHe $\mathrm{LH}$, ambos com $p<0,0 \mathrm{l}$.

\section{Discussão}

A menstruação é um sangramento genital periódico e temporário que é o fim de uma série de eventos endócrinos dinâmicos interdependentes do sistema hipotálamohipófise-ovário.

No ciclo menstrual normal, há uma produção hormonal cíclica ordenada e uma proliferação paralela do revestimento uterino no preparo para implantação do embrião. Esse ciclo dura de $2 \mathrm{I}$ a 35 dias, com 2 a 6 dias de fluxo e uma perda sanguínea média de 20-60ml. No início do ciclo menstrual, os níveis de esteróides gonadais estão baixos, os 
níveis de FSH começam a aumentar e um grupo recrutado de folículos secreta níveis crescentes de estrógeno (fase folicular) que, por sua vez, estimula a proliferação do endométrio, diminui a secreção de $\mathrm{FSH}$ e estimula a secreção de $\mathrm{LH}$ até 0 pico hipofisário de LH, com consequente ovulação 24-36 horas após. No final dessa fase, receptores induzidos pelo $\mathrm{FSH}$ e estimulados pelo LH modulam a secreção de progesterona, que aumenta subitamente após a ovulação, ao mesmo tempo em que há diminuição dos níveis de estrógeno. A ovulação dá início a chamada fase lútea, que é caracterizada por níveis elevados de progesterona e estrógeno, que desde o meio da fase lútea aumentam novamente devido à secreção do corpo lúteo. Não havendo gravidez, o corpo lúteo se extingue levando à consequente diminuição dos níveis de estrógeno e progesterona, estabelecendo o patamar para o próximo ciclo ${ }^{16,17}$.

Nosso trabalho teve como objetivo avaliar os níveis séricos de FSH, LH, PRL eE2 em mulheres com insuficiência renal em programa crônico de diálise. Como havia pacientes que menstruavam regularmente (Grupol) e pacientes amenorreicas mesmo com idade compatível com o menacme, essas últimas foram divididas em dois grupos: o Grupo II, com idade inferior a 48 anos e o Grupo III, com idade igual ou superior a 48 anos. Utilizamos esse limite para caracterizar, de modo arbitrário, as pacientes amenorreicas que provavelmente deveriam estar na menopausa, sem levar em conta, inicialmente, as dosagens séricas das gonadotropinas; tal limite, como já citado, foi estabelecido com base em trabalho epidemiológico de Halbe et al. ${ }^{16}$.

$\mathrm{Na}$ IRCT, a hiperprolactinemia é descrita como presente na maioria dos pacientes ${ }^{18}$ ou em apenas $30 \%$ deles $^{19}$, resultando predominantemente da produção aumentada, embora também haja diminuição da degradação. Os níveis de prolactina (PRL) na uremia não aumentam normalmente com a estimulação por $\mathrm{TRH}$, fenotiazidas, arginina, ou hipoglicemia insulino-induzida, e, do mesmo modo, não caem adequadamente após a administração de dopamina, L-dopa ou bromoergocriptina, embora o tratamento crônico com estaúltima possa diminuir os níveis de prolactina em pacientes com IRC ${ }^{11,18}$. Ograu da elevação dos níveis de PRL é moderado (três a seis vezes o normal) e de importância clínica incerta, sendo que graus extremos de hiperprolactinemia (acima de $100 \mathrm{ng} / \mathrm{ml}$ ) poderiam sugerir doença hipofisária concomitante ${ }^{19}$. A hiperprolactinemia correlaciona-se bem com a galactorréia, mas não com a amenorréia ${ }^{9}$ é considerada importante na infertilidade e nas disfunções sexuais, tais como diminuição de desejo e dificuldade de se obter o orgasmo que estas mulheres apresentam ${ }^{5,19,20}$. O tratamento com a bromoergocriptina é controverso, com relatos de correção dos distúrbios do sistema hipotálamo-hipofisário-gonadal| ${ }^{18} \mathrm{ere}$ latos de ausência de melhora na menstruação e na lactação ${ }^{11,19}$.

Em relação às gonadotropinas, há uma concordância razoável na literatura, com a maioria dos autores relatando níveis normais ou discretamente elevados de hormônio folículo-estimulante (FSH) e elevados de hormônio luteinizante (LH). $\mathrm{O}$ aumento dos níveis de FSH e LH, decorrente da administração do hormônio liberador de LH (LH-RH) ou clomifeno e após a menopausa, sugere que a produção, armazenamento e liberação das gonadotropinas estariam normais, refletindo uma retroalimentação negativa normal do estrógeno sobre as gonadotropinas. Ao contrário, a retroalimentação positiva do estrógeno sobre o hipotálamo, responsável pelo aumento do FSH e LH no meio do ciclo, estaria alterada por razões ainda desconhecidas. A principal consequência clínica destas alterações é que $50 \%$ das mulheres em diálise são amenorreicas. Dentre as que menstruam, muitos ciclos são anovulatórios, com fluxo irregular e tipicamente escasso, embora também possa ocorrer hipermenorréia, que aumentaria a necessidade de transfusões. A infertilidade é a regra nestas mulheres, embora possa ocorrer gravidez $8,21,18,22,23,5,24,25,19$.

Os relatos mais antigos das dosagens de estrógeno e progesterona nas mulheres em diálise mostravam diminuição desses dois hormônios e chegavam a sugerir uma insuficiência ovariana ${ }^{8,9,26,18}$, enquanto os mais recentes descrevem níveis normais de estrógeno e portanto, sugerem função ovariana intacta $^{23,24,19}$, embora haja relato de que $30 \%$ das mulheres pré-menopausadas apresentam hipoestrogenismo ${ }^{20}$.

Essas alterações hormonais não se modificam nas fases finais da IRC, ou seja, antes, nas fases iniciais ou após anos de diálise $\mathrm{e}^{27,21,28,24}$, emboraalgumas mulheres voltem a menstruar após o início da terapêutica dialítica ${ }^{29,5,30}$. 0 tipo de diálise (hemodiálise versus diálise peritoneal ambulatorial contínua) não interfere no padrão hormona| ${ }^{31}$, assim como os níveis dos diferentes hormônios não mostram diferenças significantes antes e depois da sessão de hemodiálise ${ }^{26,32}$.

No Grupo I, das pacientes que menstruam regularmente, as dosagens de $\mathrm{FSH}$ e LH encontravam-se dentro da faixa da normalidade, com exceção de uma única paciente com níveis baixos, a saber, 0,91 e 0,8 mUI/ $\mathrm{ml}$. A citada paciente tem 30 anos de idade e fez uso de anticoncepcional oral por três meses devido a um quadro de metrorragia, tendo parado com a medicação um mês antes da dosagem. Esse fato poderia explicar os níveis baixos de FSH, por um efeito de bloqueio do eixo hipotálamo-hipofisário determinado pelo contraceptivo. Neste grupo, houve concordância com a maior parte da literatura no tocante às dosagens de $\mathrm{FSH}$, que se mostram dentro da normalidade; nas dosagens de LH, embora muitos autores relatem níveis elevados ${ }^{26,23,31,36,37}$, não encontramos nenhum valor acima do considerado normal. Também aqui houve uma paciente com níveis ligeiramente diminuídos: trata-se de portadora de lúpus eritematoso sistêmico submetida a corticoterapia crônica com prednisona.

Os níveis de PRL mostraram-se elevados em todas as pacientes, assim como nos relatos de Morley et al. ${ }^{9}$, Peces et al. ${ }^{1 "}$, Schaefer et al ${ }^{12,13}$ e Koutsikos et al. ${ }^{23}$. Em cinco pacientes foram observados níveis muito mais elevados do que os $100 \mathrm{ng} / \mathrm{ml}$ referidos por Bainbridge e Lim ${ }^{19}$ e parece provável que tais níveis sejam decorrentes da associação IRC, uso de drogas do tipo alfametildopa e aminas antieméticas, que aumentam a secreção hipofisária da $\mathrm{PRL}^{5,19}$.

Em relação ao E2, a média e mediana do grupo estavam dentro do intervalo normal. Os valores normais de $\mathrm{LH}, \mathrm{FSH}$ e E2 poderiam 
sugerir eixo hipotálamo-hipofisário-gonadal íntegro, o que Kokot et al. ${ }^{15}$ sugerem ser devido à ação da EPO, mas os altos níveis de PRL das nossas pacientes não coincidem com a normalização relatada com o uso da EPO obtida por Schaefer et al. ${ }^{12,13}$.

No Grupo II, duas pacientes mostraram níveis elevados de FSH e LH: a paciente de $n^{\circ} 12$ mostrava também níveis baixos de E2, 45,9 anos de idade e não menstruava há três anos, estando em diálise há apenas meio ano; a paciente $n^{\circ} 33$, com 46,9 anos, apresentava níveis normais de $E 2$ e não menstruava desde os 42 anos de idade, estando em diálise há apenas um ano. Os altos níveis de FSH e LH vieram a sugerir que se tratavam de pacientes já menopausadas. As demais pacientes apresentaram níveis dentro da normalidade de ambos os hormônios, inclusive sem diferença estatística significante com o grupo das que menstruam. Os níveis de PRL foram, em sua maioria, elevados e também neste grupo, houve sete doentes com dosagens acima dos $100 \mathrm{ng} / \mathrm{ml}$.

Em relação às dosagens de E2 desse grupo, seis pacientes mostraram níveis diminuídos, sendo que duas delas apresentavam os maiores níveis de PRL (65I,5 e $487,3 \mathrm{ng} / \mathrm{ml}$ ) e uma terceira foi aquela paciente considerada menopausada; entre as outras três, havia uma paciente de 45,3 anos, em diálise há apenas 0,1 ano e com história de ausência de menstruação há três meses, não havendo recuperado a função menstrual até o final do estudo. As seis pacientes restantes mostravam níveis normais de estradiol. Com base em tais dados, podemos sugerir que haja integridade do eixo hipotálamo-hipofisáriogonadal, uma vez que as pacientes com hipoestrogenismo são menopausadas que foram incluídas neste grupo por terem menos de 48 anos. Ressalte-se que, semelhantemente ao grupo das mulheres que menstruam, a maior parte das pacientes (92\%) apresentava hiperprolactinemia. É interessante notar que não houve nenhuma diferença estatística significante entre 0 grupo de mulheres que menstruam e as amenorreicas com menos de 48 anos, inclusive quanto à hiperprolactinemia. Esse fato nos leva a supor que na gênese da amenorréia dessas mulheres possam participar fatores diferentes de uma deficiente reserva ovariana, como a deficiência de oligoelementos e a presença de toxinas urêmicas não identificadas, conforme sugeriram Kokot et al. ${ }^{22}$.

No Grupo III, mulheres amenorreicas com mais de 48 anos de idade, e que foram consideradas como menopausadas, houve quatro dosagens de FSH diminuídas em relação aos valores esperados para mulheres nesse período, porém as idades das pacientes (63,4 a 78,8 anos) confirmam a pósmenopausa. As demais pacientes mostravam níveis elevados de FSH conforme o esperado para mulheres menopausadas, notando-se que três delas apresentavam níveis superiores ao intervalo considerado normal. Os níveis de LH se encontravam abaixo dos níveis esperados nas mesmas pacientes que apresentavam níveis baixos de FSH. Houve, porém, seis pacientes com níveis mais elevados: duas coincidindo com altas dosagens de FSH (as de $n^{\circ} 8$ e 32 ) e as demais com níveis esperados de FSH. Todas as pacientes apresentavam níveis elevados de prolactina, com nove dosagens acima de $100 \mathrm{ng} / \mathrm{ml}$. Os níveis séricos de FSH e LH nas pacientes do Grupo III, mostraram-se compatíveis com o período da menopausa, o que não ocorreu com o grupo das mulheres amenorreicas com menos de 48 anos de idade; há uma diferença estatisticamente significante nos valores séricos das gonadotropinas $(p<0.01)$. Tal fato sugere que a amenorréia das pacientes portadoras de IRC não se deva a função ovariana deficiente, como ocorre na menopausa.

Nossos resultados mostram que os parâmetros de FSH, LH e E2 estavam dentro do limite considerado normal para mulheres sem IRC, porém, em todos os grupos, a PRL foi muito elevada em relação ao normal. Kokot et al. ${ }^{15}$ relataram diminuição dos níveis elevados de FSH e LH após tratamento com EPO, enquanto Boomer et al. ${ }^{14}$ e Lawrence et al. ${ }^{38}$ relataram que não havia qualquer alteração significante nos níveis destes hormônios ou da PRL com o tratamento pelaEPO. Como todas as nossas pacientes faziam uso de EPO, conforme indicação clínica para pacientes renais crônicas, não dispomos de meios para comparação com doentes que não fazem uso dessa medicação.

Mesmo assim, chama a atenção o fato de nossas pacientes terem níveis normais de $\mathrm{LHe}$ E2, em contraste com muitos autores ${ }^{9,5,18,26}$, assim como uma alta porcentagem de hiperprolactinemia, descrita como presente na maioria da população urêmica por Mujais et al. ${ }^{18}$, mas restrita a apenas $30 \%$ da população dialisada segundo Bainbridge e Lim ${ }^{19}$.

O fato de que os níveis de gonadotropinas encontrados foram normais nos coloca em concordância também com Strickler ${ }^{27}$, que sugere que o rim não seria o único meio de depuração das gonadotropinas já que os referidos níveis estão dentro da normalidade. Os mesmos dados, porém, estão em desacordo com Mantouvalos ${ }^{26}$, que descreveu níveis de FSH mais baixos que o normal, níveis de $\mathrm{LH}$ mais altos e de estradiol extremamente baixos, que sugeririam por sua vez comprometimento da função ovariana.

Interessante notar que, assim como Soffer ${ }^{21}$ e Jungers etal. ${ }^{29}$, nossas mulheres ditas menopausadas apresentaram valores comparáveis aos das mulheres normais, mostrando também que o limite de 48 anos utilizado por nós para classificação das amenorreicas com menos de 48 anos como pré-menopausadas e das amenorreicas com mais de 48 anos como pós-menopausadas serviu adequadamente ao seupropósito.

Acreditamos que o fato de nossas pacientes com menos de 48 anos, menstruantes ou não, apresentarem níveis de FSH, LH e estradiol compatíveis com o perfil hormonal do menacme, sugere que a reserva ovariana destas pacientes esteja preservada e que o eixo hipotálamohipofisário esteja íntegro. Consideramos que se deva buscar justificativas diferentes do comprometimento da função ovariana para explicar a ausência de menstruação em algumas pacientes com insuficiência renal em programa crônico de diálise, como a deficiência de oligoelementos e a presença de toxinas; os altos níveis de PRL encontrados poderiam exercer algum papel, mas salientamos que a hiperprolactinemia é condição que igualmente aparece nas portadoras de IRC que menstruam regularmente. 


\section{SUMMARY}

Sex hormones in wOMEn on dialysis

OBJECTIVE. The end stage renal disease has accompaniments in body systems, including hormonal changes. The present study was designed to evaluate the pattern of follicle stimulating hormone (FSH), luteinizing hormone $(L H)$, prolactin (PRL) and estradiol (E2) in 47 women with end stage renal disease (ESRD), on regular dialysis and receiving recombinant human erythropoietin (r-HuEPO).

Methods. One patient was on intermitent peritoneal dialysis, 4 on continuous ambulatory peritoneal dialysis and 42 on maintenance haemodialysis. They were divided into: Group I consisted of 10 women with regular menses, aged lesser than 48 years; Group II consisted of 15 women with amenorrhea, aged lesser than 48 years and; Group III, consisted of 22 women with amenorrhea, aged 48 years or more. Levels of FSH, LH, PRL e E2 were obtained by ELISA.

RESULTS. All groups showed FSH, LH and E2 levels in the normal range for normal women without renal failure, and there was no statistical significance in the levels of these hormones between women with regular menses or women with amenorrhea aged lesser than 48 years. The serum concentrations of PRL were above the normal range in all groups. The patients aged 48 or more showed hormonal profile consistent with post-menopausal (i.e. high FSH, high LH and low estradiol).

CONCLUSIONS. We conclude that ovarian function is intact on ESRD and we have to search for other causes for amenorrhea in women that do not have na abnormal hormonal sex profile. [Rev Assoc Med Bras 2003; 49(4): 4I8-23]

KEY WORDS: End stage renal disease. Dialysis. Sex hormones. Women. Recombinant human erythropoietin.

\section{REFERÊNCIAS}

I. Lentino RL, Leehey DJ. Infecções. In: Daugirdas JT, Ing TS, editores. Manual de diálise. $2^{\mathrm{a}}$ ed. São Paulo: Medsi Ed. Médica e Científica Ltda; 1996. p.437-8.

2. Paganini EP. Anormalidades hematológicas. In: Daugirdas JT, Ing TS, editores.. Manual de diálise. $2^{\mathrm{a}}$ ed. São Paulo: Medsi Ed. Médica e Científica Ltda; 1 996. p.4I0.

3. lanhez LE, Sabbaga E. Transplante renal. In: Cruz J, Praxedes JN, Cruz HMM, editores. Nefrologia. São Paulo: Sarvier Editora de Livros Médicos Ltda; I 994. p.229-30.
4. Klein KL, Korokawa K. Metabolic and endocrine alterations in end-stage renal failure. Postgrad Med 1978; 64(5):99- 108.

5. Lazarus JM, Hakim RM. Medical aspects of hemodialysis. In: Brenner BM, Rector FC Jr., editors. The k.idney. $4^{\text {th }}$ ed. Philadelphia: WB Saunders; 1991. p. 2250.

6. Lim VS, Auletta F, Kathpalia S, Frohman LA. Gonadal function im women with chronic renal failure: a study of the hypothalamo-pituitaryovarian axis. Proc Clin Dial Transplant Forum 1977; 7:39-47.

7. Lim VS, Kathpalia SC, Henriquez,C. Endocrine abnormalities associated with chronic renal failure. Med Clin North Am I 978; 62(6): I 34 I-6I.

8. Perez RJ, Lipner H, Abdulla N, Cicotto S, Abrams M. Menstrual dysfunction of patients undergoing chronic hemodialysis. Obstet Gynecol I978; 5 I(5):552-5.

9. MorleyJE, Distiller LA, Epstein S, Katz M, Gold C, Sagel J, et al. Menstrual disturbances in chronic renal failure. Horm Metab Res 1979; I I ( I):68-72.

10. MorleyJE, Melmed S. Gonadal dysfunction in systemic disorders. Metabolism 1979; 28(I0): $1051-73$.

II. Peces R, Horcajada C, Lopez-Novoa JM, Frutos MA, CasadoS, Hernando L. Hyperprolactinemia in chronic renal failure: impaired responsiveness to stimulation and supression. Normalization after transplantation. Nephron I 98I; 28(I): I I-6.

12. Schaefer RM, Kokot F, Wernze H, Geiger H, Heidland A. Improved sexual function in hemodialysis patients on recombinant erythropoietin: a possible role for prolactin. Clin Nephrol I989; 3 I (I): I-5.

13. Schaefer RM, Kokot F, Knerner B, Zech M, Heidland A. Normalization of serum prolactin levels in hemodialysis patients on recombinant human erythropoietin. Int J Artif Organs 1989; I2(7):445-9.

14. Bommer J, Kugel M, Schwobel B, Ritz E, Barth HP, Seeling R. Improved sexual function during recombinant human erythropoietin therapy. Nephrol Dial Transplant I 990; 5(3):204-7.

15. Kokot F, Wiecek A, Grzesczak W, Klin M. Influence of erytropoietin treatment on follitropin and lutropin response to luliberin and plasma testosterone levels in haemodialyzed patients. Nephron 1990; 56(2): I 26-9.

16. Halbe WH, Bedone AJ, Cunha DC. Controle neuroendócrino do ciclo menstrual. In: Halbe WH, editor. Tratado de ginecologia. São Paulo: Roca; 1998. p.292-304.

17. Guyton AC, Hall JE. Fisiologia feminina antes da gravidez e os hormônios femininos. In: Guyton AC, editor. Tratado de fisiologia médica. Rio de Janeiro: Guanabara Koogan; 1997. p.925-35.

18. Mujais SK, Sabatini S, Kurtzman NA. Pathophysiology of the uremic syndrome. In: Brenner BM, Rector FC Jr, editors. The kidney. $3^{\text {rd }}$ ed. Philadelphia: WB Saunders Company; 199I. p.|596-7.

19. Bainbridge T, Lim V.S. Distúrbios endócrinos. In: Daugirdas JT, Ing TS, editores. Manual de diálise $2^{\mathrm{a}}$ ed. São Paulo: Medsi Ed. Médica e Científica Ltda; I996. p.460.
20. Matusklewicz-Rowinska J, Skorzewska K, RadowickiS, SokalskiA, PrzedlackiJ, Niemczyk $S$, et al. The benefits of hormonal replacemente therapy in premenopausal women with oestrogen deficiency on haemodialysis. Nephrol Dial Transplant 1999; 14(5): I 238-43.

21 . Soffer $O$. Sexual dysfunction in chronic renal failure. South Med J 1980; 73(I2) 1599-606.

22. Kokot F, Grzeszczak W, Dulawa J. Gonadal function in patients with acute and chronic renal failure. Contrib Nephrol 1986; 50(I): 103-8.

23. Koutsikos D, Sarandakou A, Agroyannis B, Tzanatos $\mathrm{H}$, Tsoutsos D, Konstadinidou I, et al. The effect of succesful renal transplantation on hormonal status of female recipients. Ren Fail 1990; I2(2): I25-32.

24. Phocas I, Sarandakou A, Kassanos D, Rizos D, Tserkesis G, Koutsikos D. Hormonal and ultrasound characteristics of menstrual function during chronic hemodialysis and after succesful renal transplantation. Int J Gynaecol Obstet 1992; 37(I): 19-28.

25. Handelsman DJ, Dong Q. Hypothalamopituitary gonadal axis in chronic renal failure. Endocrinol Metab Clin North Am 1993; 22(I): |45-6I

26. Mantouvalos $\mathrm{H}$, Metallinos $\mathrm{C}$, Makrygiannakis A, Gouskos A. Sex hormones in women on hemodialysis. Int J Gynaecol Obstet 1984; 22(5):367-70.

27. Strickler RC, Woolever CA, Jonhson $M$, Goldstein M, Deveber G. Serum gonadotropins patterns in patients with chronic renal failure on hemodialysis. Gynecol Invest 1974;5(4): 185-98.

28. Bonomini V, Orsoni G, Stefoni S, Vangelista A. Hormonal changes in uremia. Clin Nephrol 1979; I I (6):275-80.

29. Jungers $P$, Netter AL, Pelissier C, Zingraff J, Soldat MC, Roger M, et al. Hormonal study of gonadal function in chronic hemodialysis patients. Ann Endocrinol (Paris) 1976; 37(6):505-6.

30. Otieno MR, Mcligeyo SO, Kigondu CS, Rogo KO. Menstrual disorders in patients with chronic renal failure. East Afr Med J 1993; 70(I):6-9.

31. Semple CG, Beastall GH, Henderson IS, ThomsonJÁ, KennedyAC. The pituitary-testicular axis of uraemic subjects on haemodialysis and continuous ambulatory peritoneal dialysis. Acta Endocrinol(Copenh) I982; I0I(3):464-7.

32. Chisvert Leon J, Valderrabano Quintana F. Alterations of the hypophyseal-gonadal axis in chronic renal insufficiency and after renal transplantation. An Med Interna 1991; 8(I2):587-94

33. Carlson HE, Graber ML, Gelato MC, Hershman JM. Endocrine effects of erythropoietin. Int J Artif Organs 1995; I8(6):309- 4.

34. Ramirez G, Bittle PA, Rabb HA, Ballester O, Bercu BB. Effect of haemoglobin and endogenous erytropoietin on hypothalamicpituitary-thyroidal and gonadal secretion: an analysis of anaemic (high EPO) and polycithaemic (low EPO) patients. Clin Endocrinol (Oxf) 1995; 43(2): 167-74. 
35. Siëgel S, Castellan NJ Jr. Non parametrics statistics. $2^{\text {nd }}$ ed. New York: McGraw-Hill; 1988. p.399.

36. Ferraris JR, Domene HM, Escobar ME, Caletti MG, Ramirez JA; Rivarola MA. Hormonal profile in pubertal females with chronic renal failure: before and under haemodialysis and after renal trans- plantation. Acta Endocrinol (Copenh) 1987; I I 5(3):289-96.

37. Medri G, Carellla C, Padmanabhan V, Rossi CM, Amato G, De Santo NG, et al. Pituitary glycoprotein hormones in chronic renal failure: evidence for an uncontrolled alpha-subunit release. JEndocrinol Invest 1993; 16(3): 169-74.

38. Lawrence IG, Price DE, Howlett TA, Harris
KP, Feehally J, Walls J. Erythropoietin and sexual dysfunction. Nephrol Dial Transplant 1997; I 2(4):74|-7.

Artigo recebido: I3/I I/2002 Aceito para publicação: 31/03/2003

\section{OPINIÃO DO LEITOR}

A nova Ramb abre espaço para o leitor na seção "Correspondências". Envie sua opinião ou sugestão sobre a revista ou artigos de seu interesse. Participe também enviando suas dúvidas ou sugestões sobre condutas práticas para a seção "À beira do leito".

Sua colaboração é muito importante para aprimorarmos o nosso veículo de comunicação. Rua São Carlos do Pinhal, 324 Cep: 01333-903 - São Paulo - SP - E-mail:ramb@amb.org.br 